been described with the greatest care, and they would seem identical in kind with those mentioned by Professor Trousseau as characteristic of progressive locomotor ataxy. (Trousseau's Clinical Medicine, New Sydenham Society's Translation, vol. i, p. 169), with this distinction, that in the latter they are confined to certain portions of the posterior columns of the spinal cord, whereas in insular sclerosis they occur also in the white matter of the brain. The same intimate pathological changes have also been described under the name of miliary sclerosis in chronic mania, tetanus, leukæmia, and idiocy. (See St. Bartholomew's Hospital Reports, vol. viii, on the Morbid Histology of the Spinal Cord, by W. B. Kesteven.)

It would seem, therefore, that the disease we have been considering is at present to be distinguished more clearly by its symptoms than by any very special pathology ; and perhaps, such terms as rhythmic or oscillating ataxy may hereafter prove to be more applicable than a designation derived from morbid changes, which may not prove to be distinctive.

Again, attempts have been made to explain the symptoms by the pathology - the rhythmic movements, for instance, by increased resist. ance to the nerve-current caused by actual loss of nerve-substance ; but it is a fair question whether the disease, in its earlier stages, may not be fully developed without any permanent morbid change in the nerve-fibres; whether, in fact, it may not begin as a functional disorder, resulting in local congestion, and eventually in local changes of a more permanent kind. It seems certain that locomotor ataxy, which is most closely allied to it in symptoms and pathology, may exist without any recognisable lesion other than local congestion. Such a supposition would at all events give a more hopeful aspect to the disease in its earlier stages; and it is certainly supported by a case such as I have now described, for it is difficult, if not impossible, in the face of such marked improvement, to believe that those destructive changes of nerve-tissue have been present which are found in long-standing cases of insular sclerosis, and which M. Charcot appears to consider as the very essence of the disease.

The delay which has occurred in the publication of this paper enables me to carry the history of the case a stage further, and to give some particulars of the child's present condition. Since November in last year, when the paper was read, improvement has been steady and uninterrupted. No one who saw the child now would believe that she was the apparently hopeless invalid of even a year ago. About the middle of December, she suddenly called the nurse's attention to the fact that she could read certain small print across the ward, which she had before been unable to distinguish, and since then her eyesight has been very fairly good. In March, she began to stand alone, and to walk a step or two with the help of the nurse's finger, or to climb round the beds and forms : she also tried rough worsted work, but at the expense of many pricks. She is now plump, with firm flesh and a round face expression still somewhat vacant, unless interested. The eyes have a slight cast, which the nurse assures me she had noticed before her present illness, but are otherwise perfectly normal-no nystagmus, even under excitement. There is no tremor or jerky movement of any kind. The hand is raised steadily, and grasps firmly even small objects. She feeds herself tidily with a spoon, uses her needle very fairly at worsted work, and is decidedly improved in her writing, although she dislikes practising very much. I give a specimen of one of her early attempts at her own name (Annie), and of her writing at the present time (the word " come"). Her left hand was originally most feeble, but she is

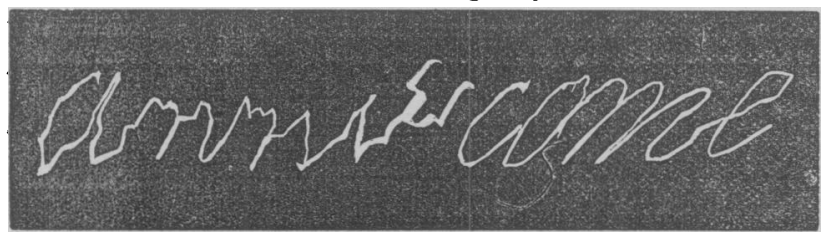

now inclined to be left-handed. When sitting, she is quite upright, with the head well up. She is not readily tired, and when with children in the garden and unobserved, she can walk or even run considerable distances without any support; yet when strangers are in the ward, she at once becomes nervous and unsteady in her gait, tottering, and glad to seek the support of the nearest bed or form. She is quick and intelligent, learns as readily by heart as the other children in the ward, and is gradually being taught to make herself generally useful.

Such is the bistory of this very interesting case up to the present date; and it seems impossible, looking to the improvement already made, to avoid anticipating, with something more than hope, a complete and well established recovery.
S M A L L.P OX.

By ROBERT BELL, M.D., F.F.P.S.G., etc., Physician to the Glasgow Ophthalmic Institution.

Although small-pox does not inspire us with the same dread that it did our forefathers before the days of the immortal Jenner, it is yet, with very good reason, a disease of which all stand in wholesome dread, for still it seems to rise as it were from its very ashes, and sweep in wild bursts of rage over whole tracts of country, attacking large numbers of the population and filling the community with alarm and dismay. It would be difficult to determine what secret causes are at work to render it capable of exerting such baneful influences, when the Vaccination Act has secured the universal introduction of cow-pox into the system of every subject of this realm. The most rational conclusion seems to be, that vaccinia produced in infancy completely resists the poison of variola for a certain period, but gradually the individual loses his insusceptibility, so that the disease, after the lapse of time, varying in different people, is not completely resisted, but regains more or less of its original power over the organism as the date of primary vaccination becomes more or less remote. Thus it becomes necessary to consider the propriety of insisting upon revaccination at intervals of eight or ten years. It is certainly a fact that, in upwards of two hundred individuals whom I vaccinated during the prevalence of small-pox in this city, and all of whom were to some extent exposed to the contagion, not one was snitten by the disease. The object of this paper, however, is not to descant upon the wonderful influence that vaccination exercises over the poison of variola, but to show huw this disease, when it makes its appearance, may be rendered as harm. less as possible.

About six years ago, when small-pox was epidemic in Glasgow, many of my patients were prostrated by it, all of whom, I am thankful to say, recovered; and I am inclined to the belief that this satisfactory result was attributable in a great measure to the plan which was adopted of treating the skin during the course of the disease.

It is only natural to conclude that the great exhaustion which ensue: in small-pox is due to the fact that the highly nervous and important as well as extensive organ, as the skin most certainly is, is in a state not only of great and intense irritation, but of almost complete inactivity as well. Now, the greatest danger of a fatal issue is generally contemporaneous with the development of the suppurative or secondary fever; and, as my method of treating the disease does away with any secondary fever, the greatest, or at least one of the greatest, sources of danger is removed. By commencing this treatment at the very beginning of the attack, the comfort of the patient is secured at once; the skin is rendered less irritable; the fever, in consequence, is kept down; the strength of the patient remains unimpaired; he is able to sleep and take nourishment, and, in short, to pass through the whole course of the attack with the minimum of discomfort. At the period when suppuration commences in the vesicles, and when otherwise a new phase of the disease would present itself, no such unhappiness is encountered, and the patient sails pleasantly through a sea of troubles, quite unconscious that he is doing so. This excellent result is due, doubtless, to the sedative effects of the remedy employed.

The plan of treatment consists in painting every part of the skin where the eruption appears with one part of carbolic acid dissolved in from eleven to fifteen parts of glycerine, and repeating the application night and morning. The urine must be watched with great care, as it often happens that the carbolic acid becomes absorbed and makes its presence known in the urine by giving the fluid a dark smoky appearance. If this be observed, the application must be made less frequently, or a weaker solution of the acid employed, as it may act too severely as a depressing agent, though I never knew this actually to result from the use of even the more concentrated solution. The employment of carbolic acid in this way has other advantages besides those already mentioned. It acts as a disinfectant, and it prevents pitting to a very great extent. The latter effect is due to the fact that suppuration does not run the same lengthened course as it does when no carbolic acid is employed, and thus the skin is not destroyed to such a depth as it would otherwise be. I could enumerate many of my patients who have suffered from what might have been called very severe attacks of small-pox, and yet now they present not the slightest trace of having had the disease.

The following cases will give some idea of the results obtained while pursuing this plan of treating the malady.

CASE I.-Mrs. T., aged 6o, a thin and delicate lady, took small-pox on November 21st, 1871, which was not only confluent, but in some parts of the body hremorrhagic. I never expected that my patient 
would recover, as, previously to this illness, she had been in a most critical state of health. Every portion of the body where the eruption made its appearance was painted over with a solution of one part of carbolic acid in twelve of glycerine. Immediately the great distress produced by the eruption was relieved, and was prevented from returning by the application being repeated night and morning. The rest of the treatment consisted in supplying plenty of fresh air and a simple yet nourishing diet. Chlorate of potash in solution was given as a drink, and the bowels were kept moving by a mild laxative given when required; and the patient passed through the whole course of the disease without an unfavourable symptom. There was no itching of the skin, and there was no secondary fever; indeed, there was no fever at all after the first application of the carbolic acid and glycerine. The patient slept well, and took her food with a relish. Within a year after her recovery, it was almost impossible to find any traces of the disease, so completely had the tendency to pitting been overcome.

CASE II.-Mrs. Y., aged 32, a strong and stoutly made lady, was confined of a healthy boy on December 7 th, 1871 , and on the day following was attacked by confluent small-pox. Under the circumstances, the greatest danger was, of course, to be apprehended, and I was, therefore, exceedingly anxious. The same treatment as in Case I was employed, and with the like satisfactory results, with the one exception that, at this date, very slight pitting can be perceived, if looked for. The baby was vaccinated before it was twenty-four hours old, and it did not take small-pox.

One more case will suffice to show that this treatment is deserving of a more extensive trial. On December 2oth, 1872, I was asked to take charge of two ladies, mother and daughter, suffering from small-pox, their own medical man declining to attend. I found the mother prostrated by an attack of confluent small-pox, and her daughter suffering from the same disease, but of the discrete variety. The features in the elder patient were quite obliterated. The same treatment was adopted in .both cases, with the effect of giving almost instant relief. As the disease held on in its course, the last named patient showed slight symptoms of prostration; and, although I was not apprehensive myself, I thought it better to have a consultation with the gentleman who then had charge of the Fever Hospital in this city. He took a very unfavourable view of the patient's condition, and gave it as his opinion that the case would probably terminate fatally when the secondary fever set in; but, as this symptom never showed itself, this danger was avoided, and my patient made a rapid recovery. It is now impossible to detect any disfigurement from pitting.

\section{CASE OF POISONING BY BELLADONNA LINIMENT.*}

By JOHN MEREDITH, M.D., Wellington, Somerset.

IN September I875, I gave a patient, a lady aged between fifty and sixty years, four ounces of methylated belladonna liniment for the relief of some muscular pains from which she was suffering. I gave her also a bottle of medicine to be taken at intervals. The liniment was properly marked, and with directions for use.

In a few days afterwards (on October Ist), I was called to see her between seven and eight o'clock in the morning, the messenger saying she had taken a dose of the wrong medicine in mistake, and was very ill. A little before seven o'clock that morning, she went to her medicinebottle, as she imagined, and took a tablespoonful of the mixture, walked out of her room and downstairs, and mentioned to some one there that her medicine had a curious taste. "In about five minutes", to use her own words, she "felt queer about her eyes, then sturidlike" in her head ; felt she was losing the power of standing on her legs. She managed, however, to get back to her room, and there discovered what she had done, and told one of the servants of it. She took some" pills she had by her "to carry the thing off", as she put it, then added she should soon die. She became helpless and quite unconscious.

Before she reached this state, some salt and water were given to her, with the view of inclucing vomiting, but without the desired result. I cannot say precisely how soon after taking the poison the unconscious state was established, but it was in much less than an hour; for it was about this time when I reached the house and saw the patient. I tried to get her to swallow some mustard and water, but failed. She could swallow only a few drops of water, and very soon she became unable to do this. She could not speak; only numbled inces. santly some inarticulate words, which were only audible at times.

* Read before the West Somerset Branch.
She was put into bed, and there sat propped up by pillows, and had a vacant drunken look; moved her hands about, sorting and catching at imaginary things; being in this respect very much like a tailor who was poisoned by belladonna, and who, according to the report of his case, "sat for hours moving his hands and arms as if sewing and his lips as if talking, but without uttering a word". In short, she was in a state of somnambulism, barring the ambulation; for in this, as in similar cases recorded, there was no power over the lower extremities, while the upper ones moved about with comparative freedom.

The other characteristic features of the case at this stage were the widely dilated pupils, and a diffuse scarlet rash, which was out on the neck and upper part of the chest, and also on the hands and forearms. I did not examine other parts of the body; if I had, most likely I should have found it equally conspicuous all over, showing how general was the paralysis of the vaso-motor nerves throughout the cuticular system.

At this stage and very soon after my arrival, the all-important question, whether I thought the patient would recover, was put to me. To this, I replied at once that I believed she would. I felt justified in saying this, because fatal poisoning by belladonna is rare, but chiefly from my recollection of a case of atropia-poisoning which was reported some years ago, where the symptoms described were very similar to those of the case before me. I need hardly say how satisfactory it was to be able to give an assurance of this sort in a house where all were in consternation, and visions of "crowner's quest", as the master of it put it, were looming in apparently close proximity.

The rash was not of long duration; it soon began to fade, and in four hours was hardly perceptible anywhere. The pulse was of fair strength all through : above 80 , but not once up to I00. The respirations were normal, not markedly accelerated. Eight hours after taking the liniment, i.e., at 3 P.M., slight signs of recovering were observed. The patient was able to swallow a little coffee. She had passed no urine up to this time. An enema of warm water with some spirits of turpentine was given, and was retained. The bowels appeared also to have been paralysed for the time. At 9 P.M., the improvement was decided. She was able to swallow and speak a little, and passed urine; but the bowels had not acted, nor was there any sickness or nausea. Next day, when I called to see her-that was about twenty-six hours after the accident-the patient expressed herself as "feeling all right"; that, however, was not strictly the case with her. She had passed the night in a similar way to which she had passed the preceding day : restless, looking and acting as if she would go off to sleep, but never really doing so.

She remembered nothing of what had happened to her from the time she took the salt and water on the morning of the rst until about 5 o'clock of the 2 gd. For about twenty-two hours, her life was a blank to her. Her condition on the morning of the and was as follows. Her pupils were widely dilated, and she saw persons and things double, and at times saw imaginary persons and objects about her; her hands felt moist, as did also her feet, and she could not stand; but she was able to move her arms about all right. The bowels recovered their power and acted during the morning. On the third day, she was much better, only her sight was still dim and she felt very unsteady on her feet.

I should mention that all through there was a good deal of distress about the throat; it was one of the first things experienced, and the discomfort lasted, to a certain extent, for many days. The patient eventually recovered completely ; but, for several days, she felt she was not her ordinary self : could not remember how to do the simplest thing; had forgotten the way; had to ponder over the way an article of clothing should be fastened on, etc.

Nothing was done in the way of special treatment beyond keeping close watch over the case, and placing the patient in as comfortable a position in bed as was practicable.

I judge that about four drachms of the liniment were swallowed, equivalent to about twelve grains of camphor, besides the macerated juice of about half an ounce of belladonna-root, in addition to the spirits forming the liniment. This manifestly is an effective way of taking belladonna, and a way that brings out its action speedily. How far this action of belladonna was affected by the camphor I cannot say. The physiological effects of camphor are not, as far as I have been able to learn, clearly known. There is something of caprice attributed to them. The truth, after all, lies, I take it, in the fact that the properties of the drug have not been sufficiently investigated.

One of the acknowledged actions of camphor in considerable doses is to depress the respiration and the action of the heart; while atropia quickens respiration in an extraordinary manner. Such being the case, the two drugs taken at the same time would modify the 